\title{
Brittle and Ductile Crack-tip Behavior in Magnesium
}

\author{
Z. Wu $u^{\mathrm{a}, \mathrm{b}, *}, \mathrm{~W} \cdot \mathrm{A} \cdot$ Curtin $^{\mathrm{a}}$ \\ ${ }^{a}$ Institute of Mechanical Engineering, École Polytechnique Fédérale de Lausanne, Lausanne \\ CH-1015, Switzerland \\ ${ }^{b}$ Institute of High Performance Computing, 1 Fusionopolis Way, \#16-16, Connexis, Singapore \\ 138632, Singapore
}

\begin{abstract}
The competition between dislocation emission and cleavage at a crack tip plays an important role in governing the intrinsic fracture behavior of crystalline materials. This competition is not well understood in magnesium, which has many different combinations of cleavage planes and dislocation slip systems. Here, using both anisotropic linear elastic fracture mechanics theory and atomistic simulations, the emission/cleavage competition in magnesium is evaluated for a comprehensive set of crack orientations and crack tip geometries under mode I crack tip stress intensity loading at $\mathrm{T}=0 \mathrm{~K}$. Both theory and simulation show that cleavage is favored in most crack orientations, including basal plane cracks, tensile twin and basalprismatic plane interface cracks. Initial slight crack tip blunting does not significantly change the behavior. These results suggest that magnesium has extremely low intrinsic fracture toughness, consistent with observations of many different cleavage-like planes in low-temperature fracture experiments. Based on $\mathrm{T}=0 \mathrm{~K}$ properties, obtaining a more-ductile material via crack tip dislocation emission on the pyramidal and basal slip systems would require substantial and moderate reductions of the unstable stacking fault energy ( $\sim 50 \%$ and $\sim 20 \%$, respectively) to be achieved at finite temperatures and/or via alloying.
\end{abstract}

Keywords: Magnesium, Cracks, Dislocations, Fracture, Molecular Dynamics Simulations

\footnotetext{
${ }^{*}$ Corresponding author. Institute of Mechanical Engineering, École Polytechnique Fédérale de Lausanne, Lausanne CH-1015, Switzerland

Email address: zhaoxuan.wu@epfl.ch (Z. Wu)
} 


\section{Introduction}

Pure magnesium $(\mathrm{Mg})$ has relatively low ductility and fracture toughness as compared to face-centred cubic (fcc) and body-centred cubic (bcc) metals. Both its low ductility and low fracture toughness are detrimental to its application as a light-weight, structure material. The low ductility of $\mathrm{Mg}$ is often attributed to its hexagonal close-packed (hcp) crystal structure and associated strong plastic anisotropy. The low fracture toughness is related to its limited energy dissipation through dislocation plasticity near cracks or at crack tips. In the near field of cracks, dislocation plasticity can change the elastic field, shield/anti-shield crack tips, dissipate energy and provide additional toughening. Plasticity depends on not only the intrinsic material properties and applied loads, but also on some extrinsic factors such as pre-existing dislocations, dislocation sources, grain and interface boundaries. Nonetheless, the intrinsic fracture toughness of $\mathrm{Mg}$, like many other crystalline materials, remains important and is governed by the competition between dislocation emission and cleavage at crack tips. While this crack-tip competition is well-established theoretically $[1,2,3]$, and in atomistic simulations $[4,5,6]$ for fcc metals, there has been no systematic study of hcp $\mathrm{Mg}$.

Unlike fcc metals with only one type of dominant and well-understood dislocation slip system, Mg has several strongly anisotropic slip systems with highly complex dislocation core structures and also multiple possible cleavage planes [7]. The competition between dislocation emission and cleavage is thus more complex and strongly dependent on the crystallographic orientations of cracks. Given accurate elastic constants, surface energies and the unstable stacking fault energies for the various slip systems, it is straightforward to analyze the crack-tip competition using linear elastic fracture mechanics (LEFM) theory for Griffith cleavage [1] and Rice's criterion [2] for dislocation nucleation within Peierls framework. Theoretical predictions are now providing some of the essential atomistic material properties for $\mathrm{Mg}$, such as the various surface energies, dislocation core structures, and unstable stacking fault energies $[8,9,10]$, so that predictions of crack tip response can be made. However, the assumptions and approximations used in the theory, the discrete nature of the atomic system, crack surface stresses, crack tip geometry, and other effects could shift the competition relative to the theory predictions. Atomistic simulations are thus useful to verify the LEFM predictions, and also necessary to reveal more-detailed insights at the atomic level. However, atomistic simulations are only viable if the underlying interatomic potential accurately reproduces the necessary material properties and also dislocation core structures for the entire range of slip systems. We have recently developed such 
an interatomic potential [11] for $\mathrm{Mg}$, and this now enables us to carry out a systematic study of the crack tip cleavage/emission competition in $\mathrm{Mg}$ and thus to provide fundamental understanding of the intrinsic fracture properties of this material.

In this work, we study the crack tip competition in $\mathrm{Mg}$ using both anisotropic LEFM theory and atomistic simulations with a modified embedded-atom method (MEAM) interatomic potential specifically fit for dislocation, plasticity and fracture properties. In particular, we investigate the dislocation emission and cleavage competition at semi-infinite crack tips loaded under mode I crack tip stress intensity factor $K_{I}$. We examine atomically sharp cracks in both LEFM theory and atomistic simulations, and slightly-blunted cracks in atomistic simulations only. We consider 9 crack orientations favoring different types of dislocation emissions and cleavage planes. For atomically sharp cracks, the theory predicts that cleavage is strongly favored for 3 crack orientations and slightly favored in 2 crack orientations while dislocation emission is strongly favored in only 1 crack orientation and slightly favored in 3 crack orientations. The stress intensity factors for cleavage are found to be comparable for all the different crystallographic planes. In the atomistic simulations of sharp cracks, crack advancement by cleavage is observed not only in the 5 cases predicted to favor cleavage, but also in the 3 cases predicted to slightly favor dislocation emissions, resulting in a total of 8 out of 9 cases exhibiting cleavage. The only simulation showing dislocation emission is the case that strongly favors emission in the LEFM prediction. In simulations of cracks with slightly blunted tip geometries, the initial response shifts from cleavage to dislocation emission in 3 cases where the competitions are predicted to be close. For the remaining cases, blunting neither changes the dominant deformation behavior nor has significant effects on increasing the fracture toughness. Overall, the cleavage-dominant behavior at crack tips confirms the extreme brittleness and low fracture toughness of $\mathrm{Mg}$, consistent with broad material science conceptions. Cleavages on different planes at comparable stress intensity factors also suggest multiple cleavages, especially at low temperatures, and this is in agreement with recent experimental results showing multiple cleavage planes on $\mathrm{Mg}$ fracture surfaces [7].

The remainder of this paper is organized as follows. In Section 2, we introduce the methods used for LEFM analysis, where anisotropic linear elastic solutions for sharp cracks in homogeneous media and special bimaterials with non-oscillatory elastic solutions are briefly presented. In Section 3, we describe the atomistic models and methods used in the simulations. In Section 4, we present the results of theory predictions and simulations. Critical stress intensity factors for Griffith 
cleavage and dislocation emission are calculated and compared with their respective simulation result for 9 different crack orientations. Section 5 contains further discussion and our conclusions.

\section{Cracks in anisotropic linear elastic media}

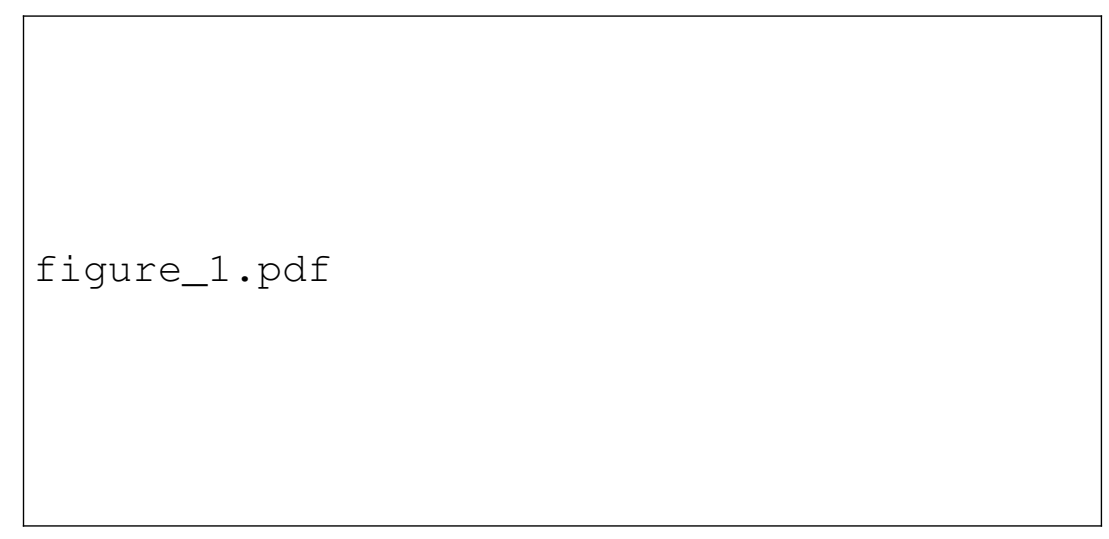

Figure 1: Schematic diagram of crack geometry and coordinate systems.

In the LEFM analysis, the elastic field at a sharp crack is solved for (i) the energy release rate, from which is calculated the critical stress intensity factor for Griffith cleavage, (ii) the elastic stress field, from which is calculated the critical stress intensity for dislocation emission, and (iii) the displacement field, which is used to apply the proper loading of cracks in the atomistic simulations. Although well-established in the literature (e.g. Reference [12] by Ting), for completeness we briefly present the totality of needed LEFM results for sharp cracks in anisotropic media.

We use a fixed Cartesian coordinate system $x_{i}$ as shown in Fig. 1 (a) and adopt a summation convention where repeated Roman indices imply summation and a comma denotes differentiation with respect to the coordinate variable $x_{i}$. The components of the displacement, stress, strain, and stiffness tensors are denoted by $u_{i}, \sigma_{i j}, \epsilon_{i j}=\left(u_{i, j}+u_{j, i}\right) / 2$, and $C_{i j k l}$, respectively. We consider anisotropic linear elastic media under plane-strain, infinitesimal deformation, i.e., $u_{k, 3}=0$ and $\epsilon_{33}=0$. Under these assumptions, the elastic field around a crack of length $2 a$ loaded by uniform tractions $\mathbf{t}_{\Gamma}$ and $-\mathbf{t}_{\Gamma}$ applied on the $\pm x_{2}$ surfaces at infinity is

$$
\mathbf{u}=\operatorname{Re}\left\{\mathbf{A}\left\langle f\left(z_{\alpha}\right)\right\rangle \mathbf{B}^{-1}\right\} \mathbf{t}_{\Gamma} \text { and } \boldsymbol{\psi}=\operatorname{Re}\left\{\mathbf{B}\left\langle f\left(z_{\alpha}\right)\right\rangle \mathbf{B}^{-1}\right\} \mathbf{t}_{\Gamma}
$$


where

$$
\begin{aligned}
& \left\langle f\left(z_{\alpha}\right)\right\rangle=\operatorname{diag}\left[f\left(z_{1}\right), f\left(z_{2}\right), f\left(z_{3}\right)\right] \\
& f(z)=\sqrt{z^{2}-a^{2}}-z, z=x_{1}+p x_{2}
\end{aligned}
$$

and $\mathbf{A}$ and $\mathbf{B}$ are the $3 \times 3$ normalized complex matrices, $\boldsymbol{\psi}$ is a stress function, and $p$ is one of the eigenvalues defined in the Stroh formalism assuming all the eigenvalues are distinct (for details, see Appendix). The explicit expression for the stress field is

$$
\begin{aligned}
& {\left[\sigma_{11}, \sigma_{21}, \sigma_{31}\right]^{T}=\mathbf{t}_{1}=-\boldsymbol{\psi}_{, 2}=-\operatorname{Re}\left\{\mathbf{B}\left\langle f\left(z_{\alpha}\right)_{, 2}\right\rangle \mathbf{B}^{-1}\right\} \mathbf{t}_{\Gamma}} \\
& {\left[\sigma_{12}, \sigma_{22}, \sigma_{32}\right]^{T}=\mathbf{t}_{2}=-\boldsymbol{\psi}_{, 1}=-\operatorname{Re}\left\{\mathbf{B}\left\langle f\left(z_{\alpha}\right)_{, 1}\right\rangle \mathbf{B}^{-1}\right\} \mathbf{t}_{\Gamma}}
\end{aligned}
$$

where

$$
\begin{aligned}
& f(z)_{, 1}=\frac{z}{\sqrt{z^{2}-a^{2}}}-1 \\
& f(z)_{, 2}=\frac{p z}{\sqrt{z^{2}-a^{2}}}-1
\end{aligned}
$$

In the polar coordinate $(r, \theta)$ with origin at the crack tip $\left(x_{1}=a, x_{2}=0\right)$ and $\theta=0$ along the crack plane,

$$
z=r(\cos \theta+p \sin \theta)+a
$$

and the asymptotic displacement field near the crack tip is given by the asymptotic limit of Eqn. 1 as $r \rightarrow 0$, i.e.,

$$
\mathbf{u}=\sqrt{2 a r} \operatorname{Re}\left\{\mathbf{A}\left\langle\sqrt{\cos \theta+p_{\alpha} \sin \theta}\right\rangle \mathbf{B}^{-1}\right\} \mathbf{t}_{0}
$$

and the singular stress field at the crack tip is given by the asymptotic limit of Eqn. 4 as $r \rightarrow 0$, i.e.,

$$
\begin{aligned}
& \mathbf{t}_{1}=-\sqrt{\frac{a}{2 r}} \operatorname{Re}\left\{\mathbf{B}\left\langle\frac{p_{\alpha}}{\sqrt{\cos \theta+p_{\alpha} \sin \theta}}\right\rangle \mathbf{B}^{-1}\right\} \mathbf{t}_{\Gamma} \\
& \mathbf{t}_{2}=-\sqrt{\frac{a}{2 r}} \operatorname{Re}\left\{\mathbf{B}\left\langle\frac{1}{\sqrt{\cos \theta+p_{\alpha} \sin \theta}}\right\rangle \mathbf{B}^{-1}\right\} \mathbf{t}_{\Gamma}
\end{aligned}
$$

The stress intensity factor is thus given by

$$
\mathbf{k}=\sqrt{\pi a} \mathbf{t}_{\Gamma}
$$


and the strain energy release rate $G$ with respect to change in crack length is

$$
G=\mathbf{k}^{T} \frac{1}{2} \mathbf{L}^{-1} \mathbf{k}
$$

where

$$
\mathbf{L}^{-1}=\operatorname{Re}\left\{i \mathbf{A B}^{-1}\right\}
$$

For cracks at the interface of anisotropic bimaterials, the elastic solution in Eqn. 1 can also be applied to both materials with their respective elastic constants and the resulting solution is non-oscillatory $[13,14,12]$ provided that

$$
\mathbf{W} \mathbf{t}_{\Gamma}=\left(\mathbf{S}_{1} \mathbf{L}_{1}^{-1}-\mathbf{S}_{2} \mathbf{L}_{2}^{-1}\right) \mathbf{t}_{\Gamma}=\mathbf{0}
$$

where the subscripts $(1,2)$ in the above matrices denotes material 1 and 2 and

$$
\mathbf{S}=i\left(2 \mathbf{A B}^{T}-\mathbf{I}\right)
$$

For such interface cracks, the strain energy release rate with respect to change in crack length is

$$
G=\mathbf{k}^{T} \frac{1}{4} \mathbf{D k}
$$

where

$$
\mathbf{D}=\mathbf{L}_{1}^{-1}+\mathbf{L}_{2}^{-1}
$$

For atomically sharp, semi-infinite cracks in homogeneous and bimaterial interfaces with non-oscillatory linear elastic solutions, the strain energy release rate for crack advancement can be written as

$$
G=\mathbf{k}^{T} \mathbf{\Lambda} \mathbf{k}
$$

where

$$
\Lambda= \begin{cases}\frac{1}{2} \mathbf{L}^{-1}, & \text { for homogeneous media } \\ \frac{1}{4} \mathbf{D}, & \text { at bimaterial interface }\end{cases}
$$

and the critical stress intensity $\mathbf{k}$ for Griffith cleavage can be found when

$$
G= \begin{cases}2 \gamma_{s}, & \text { for homogeneous media } \\ 2 \gamma_{s}-\gamma_{i}, & \text { at bimaterial interface }\end{cases}
$$


where $\gamma_{s}$ is the surface energy of the cleavage plane and $\gamma_{i}$ is the interface energy. For example, for cracks at bimaterial interfaces,

$$
G=\frac{1}{4} \mathbf{k}^{T} \mathbf{D k}=2 \gamma_{s}-\gamma_{i}
$$

Under mode I loading, the critical stress intensity factor for Griffith cleavage is $\mathbf{k}=\left\{0, K_{I c}, 0\right\}$ and can be solved in the above equation as

$$
G=\frac{1}{4} D_{22} K_{I c}^{2}=2 \gamma_{s}-\gamma_{i}
$$

i.e.,

$$
K_{I c}=2 \sqrt{\frac{2 \gamma_{s}-\gamma_{i}}{D_{22}}}
$$

The critical stress intensity factor for dislocation nucleation from the crack tip can be computed based on the theory of Rice [2] extended to anisotropic elasticity by Sun et al [15] as

$$
\mathbf{s}(\phi) \cdot \mathbf{k}^{e f f}=\sqrt{\gamma_{u s} O(\phi, \theta)}
$$

where $\theta$ is the angle between the crack plane and slip plane, $\phi$ is the angle between the dislocation Burgers vector and crack front direction in the slip plane (see Fig. 1 (b)), and $\gamma_{u s}$ is the unstable stacking fault energy. $\mathbf{k}^{\text {eff }}$ is the effective stress intensity factor as proposed by Rice [2],

$$
\mathbf{k}^{e f f}=\mathbf{F}(\theta) \cdot \mathbf{k}
$$

where $\mathbf{F}(\theta)$ is the angular-dependent matrix in the stress function (Eqn. 8) resolved in the slip plane. $\mathbf{s}(\phi)$ is the slip vector in the constrained path approximation [2], i.e.,

$$
\mathbf{s}(\phi)=(\cos \phi, 0, \sin \phi)
$$

and

$$
o(\phi, \theta)=s_{i}(\phi) \Lambda_{i j}^{(\theta)^{-1}} s_{j}(\phi)
$$

where

$$
\Lambda_{i j}^{(\theta)}=\Omega_{i k} \Lambda_{k l} \Omega_{l j}
$$

and

$$
\boldsymbol{\Omega}=\left[\begin{array}{ccc}
\cos \theta & \sin \theta & 0 \\
-\sin \theta & \cos \theta & 0 \\
0 & 0 & 1
\end{array}\right]
$$

Eqns. 18 and 22 allow us to calculate the critical stress intensity for Griffith cleavage $\left(K_{I c}\right)$ and dislocation emission $\left(K_{I e}\right)$, respectively. 


\section{Crack geometries and simulation models}

The above LEFM analysis can be applied to any crack orientation in single crystals and some special interface cracks in bicrystals. For Mg, earlier studies based on DFT [8, 9, 10] and MEAM [11] show that the surface energies of several low index planes are similar while the unstable stacking fault energies of different slip systems are starkly different. Experimental work by Bhattacharya et al [7] also observed multiple cleavage planes (basal and prismatic) on fracture surfaces of Mg at low temperatures. All these suggest that the crack tip competition in $\mathrm{Mg}$ is orientation-dependent and a large set of crack orientations should be examined. Therefore, we consider a total of 9 crack orientations as shown in Fig. 2 together with their relevant slip systems for dislocation emission. We consider dislocation emission on the slip planes that intersect the crack front along the entire crack line. Fig. 2 (a-f) correspond to cracks along the basal, prismatic, pyramidal I and II planes with different crack front orientations favoring different dislocation nucleations. Cracks on twin interfaces may also be important, as suggested by recent experiments where twin related microcracks were observed in scanning electron microscope (SEM) micrograph of Mg alloy during uniaxial and controlled triaxial loadings [16] and three-point bending test of single crystal Mg [17]. Therefore, we examine two cracks (Fig. 2 (g,h)) on the two sides of the $\{10 \overline{1} 2\}\langle\overline{1} 011\rangle$ tensile twin interface; one crack is often considered to be intrinsically more ductile than the other [18]. The last case (Fig. 2 (i)) is a crack along a basal-prismatic (BP) interface, which is a frequently-observed interface during twin growth in atomistic simulations [19, 20, 21, 22, 23] and in recent experiments $[24,25]$. Fortunately, these three bicrystal cases satisfy the non-oscillatory conditions for interface cracks. In fact, because of the symmetry of the hcp lattice, $\mathrm{Mg}$ bicrystals formed at tilt boundaries where the tilt axis lies in the basal plane or is the c-axis satisfy the decoupling condition (in-plane and anti-plane deformations of each crystal are decoupled) and the interface crack has non-oscillatory elastic solutions $[13,14]$.

The LEFM analysis in the previous section can be applied to each of the above cases to compute the critical stress intensities for Griffith cleavage and dislocation emission. To verify such predictions, we also examine each case using atomistic simulations for semi-infinite crack configurations. For each case, the simulation cell has dimensions $(x \times y \times z)$ of $\sim 240 \mathrm{~nm} \times 240 \mathrm{~nm} \times 2 \mathrm{~nm}$ with periodic boundary conditions in the $z$ direction parallel to the crack front. The crack is loaded by imposing the anisotropic elastic displacement field solution under mode I crack tip stress intensity factor $K_{I}$. Atoms within $2 r_{c}(2 \times$ cut off distance of 
This is a pre-print of the following article: Wu, Z.; Curtin, W. A. Acta Mater. 2015, 88, 1-12.. The formal publication is available at http://dx.doi.org/10.1016/j.actamat.2015.01.023

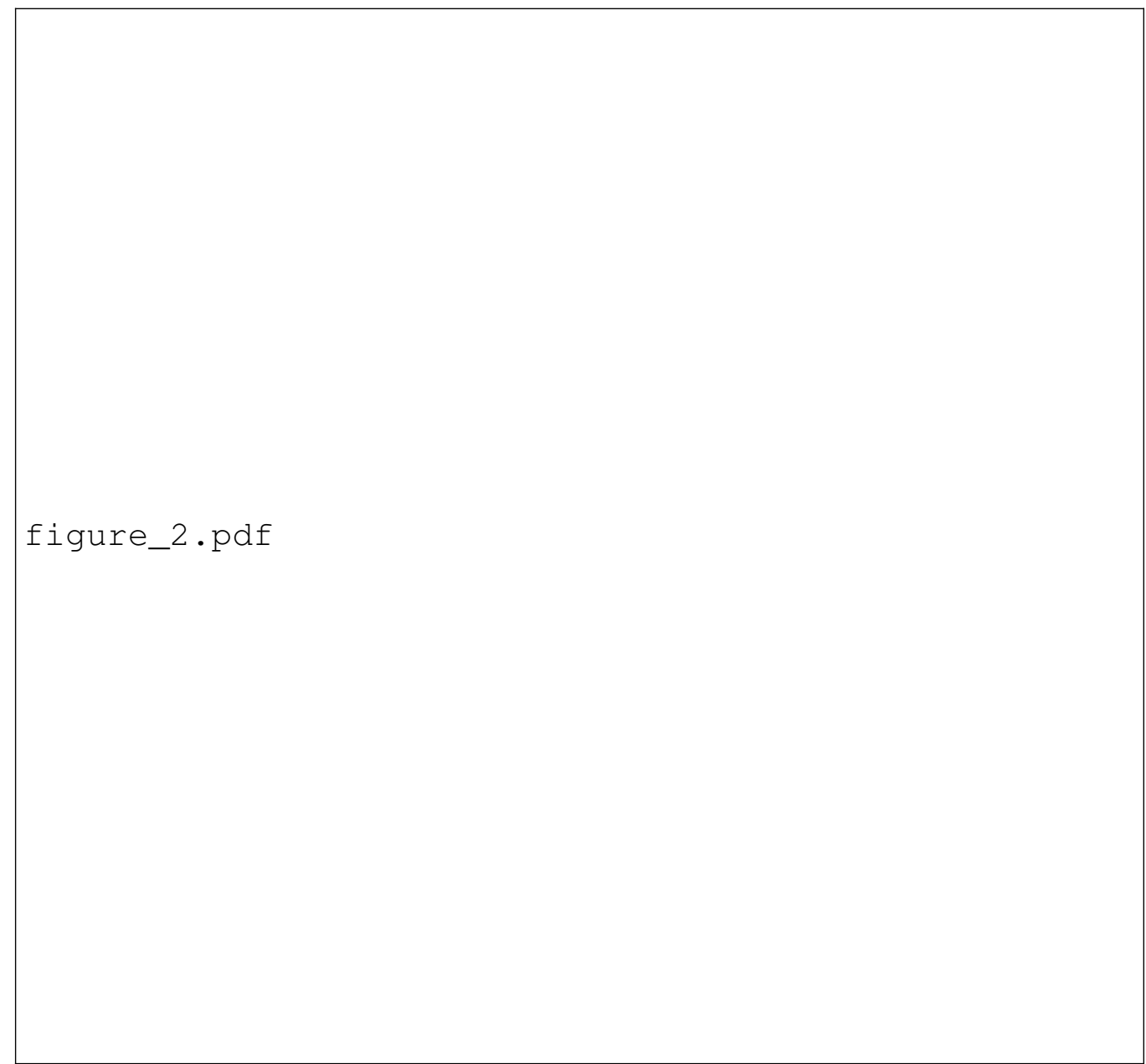

Figure 2: Schematic diagram of 9 crack orientations and relevant dislocation slip systems (slip planes and Burgers vectors for partial and full dislocations) in $\mathrm{Mg}$. 
the potential) from the boundary are fixed at the elastic displacement solution to set the boundary conditions. The remaining atoms are then relaxed by minimizing the total energy using a conjugate gradient algorithm.

Atomically sharp cracks can be unstable to crack closure/advancement when loaded below/above the stress intensity factor for Griffith cleavage. Thus, it is difficult to create a truly atomically-sharp crack in a mechanically stable configuration. To address this issue, one can remove a few layers of atoms or artificially switch off interatomic interactions between atoms on either side of the crack surface so that traction free crack surface boundary conditions are created; neither is ideal. However, we note that when subject to small perturbations around $K_{I c}$, the crack tip only moves a small distance $(\sim 1 \mathrm{~nm})$, despite the fact that the boundary conditions are applied at more than $100 \mathrm{~nm}$ away from the tip. We therefore use truly sharp crack configurations (no removed atoms or removed interactions) by starting simulations at an initial $K_{I}$ slightly below (1-3\%) the computed $K_{I c}$ and then increasing the load by small increments $\left(0.01 \mathrm{MPa} \mathrm{m}^{1 / 2}\right.$, less than $5 \%$ of $K_{I c}$ ) followed by energy minimization. For slighted blunted cracks, we intentionally remove several layers of atoms to create the desired crack tip radius, and here study tip radii of $\sim 0.5 \mathrm{~nm}$ and $1 \mathrm{~nm}$.

In this work, the molecular static simulations are performed using the Largescale Atomic/Molecular Massively Parallel Simulator (LAMMPS) [26] and atomic configurations are visualized using the Open Visualization Tool (OVITO) [27]. Interactions between $\mathrm{Mg}$ atoms are described by an MEAM-type [28, 29] potential parametrized by $\mathrm{Wu}$ et al [11]. This potential, based on earlier work of Kim et al [30], reproduces all the necessary mechanical properties for plastic deformation including the various generalized stacking fault energy surfaces, dislocations core structures, Peierls stresses, surface energies, and basal plane cohesive strength. As evident from the theory, accurate surface and unstable stacking fault energies are important for the crack tip competition, while realistic dislocation core structures and Peierls stresses ensure proper dislocation nucleation and subsequent glide.

\section{Results}

To apply the LEFM analysis on crack tip competitions in $\mathrm{Mg}$, we use the elastic constants, surface energies, and unstable stacking fault energies calculated based on the MEAM potential [11] as material parameters in the calculations of the critical stress intensities for Griffith cleavage and dislocation emission. Previous DFT and MEAM calculations [11] on generalized stacking fault energies and dislocation core structures suggest that partial dislocations on the basal and 
pyramidal I and II planes will nucleate first, in preference to full dislocations. Therefore, we use the unstable stacking fault energy corresponding to the nucleation of the leading partial as the appropriate $\gamma_{u s}$ in the theory along with the partial Burgers vector. The results of the LEFM analysis are summarized in Table 1. For cases with multiple possible dislocation slip systems, the slip system with the lowest $K_{I e}$ is presented.

\begin{tabular}{|c|c|c|c|c|c|c|c|c|c|c|}
\hline \multirow[t]{2}{*}{ Crack } & \multirow{2}{*}{$\begin{array}{l}\text { Orientation } \\
(\mathbf{n})[\mathbf{l}]\end{array}$} & \multirow[t]{2}{*}{ Slip plane } & \multirow{2}{*}{\multicolumn{2}{|c|}{$\begin{array}{rr}\gamma_{s} & \gamma_{u s} \\
\left(\mathrm{~mJ} \mathrm{~m}^{-2}\right) \\
\end{array}$}} & \multirow{2}{*}{\multicolumn{2}{|c|}{$\begin{array}{ll}K_{I c} & K_{I e} \\
\left(\mathrm{MPa} \mathrm{m}^{1 / 2}\right)\end{array}$}} & \multicolumn{2}{|c|}{ Sharp crack } & \multicolumn{2}{|c|}{ Blunted crack (tip radius) } \\
\hline & & & & & & & Predicted & Observed & $0.5 \mathrm{~nm}$ & $1 \mathrm{~nm}$ \\
\hline Basal & $\begin{array}{l}(0001)[1 \overline{2} 10] \\
(0001)[\overline{1} 010]\end{array}$ & $\begin{array}{l}\text { Pyramidal I } \\
\text { Pyramidal II }\end{array}$ & 568 & $\begin{array}{l}319 \\
295\end{array}$ & 0.255 & $\begin{array}{l}0.351 \\
0.333\end{array}$ & $\begin{array}{l}\text { cleavage } \\
\text { cleavage }\end{array}$ & $\begin{array}{l}\text { cleavage } \\
\text { cleavage }\end{array}$ & $\begin{array}{c}\text { cleavage } \\
\langle\mathbf{c}+\mathbf{a}\rangle, \text { cleavage }\end{array}$ & $\begin{array}{c}\text { cleavage } \\
\langle\mathbf{c}+\mathbf{a}\rangle, \text { cleavage }\end{array}$ \\
\hline Prism & $\begin{array}{l}(\overline{1} 010)[1 \overline{2} 10] \\
(\overline{1} 2 \overline{1} 0)[\overline{1} 010]\end{array}$ & $\begin{array}{l}\text { basal } \\
\text { basal }\end{array}$ & $\begin{array}{l}582 \\
651\end{array}$ & $\begin{array}{l}125 \\
125\end{array}$ & $\begin{array}{l}0.252 \\
0.267\end{array}$ & $\begin{array}{l}0.236 \\
0.262\end{array}$ & $\begin{array}{l}\text { basal }\langle\mathbf{a}\rangle \\
\text { basal }\langle\mathbf{a}\rangle\end{array}$ & $\begin{array}{l}\text { cleavage } \\
\text { cleavage }\end{array}$ & $\begin{array}{c}\text { cleavage } \\
\text { basal }\langle\mathbf{a}\rangle \text {, cleavage }\end{array}$ & $\begin{array}{l}\text { basal }\langle\mathbf{a}\rangle \text {, cleavage } \\
\text { basal }\langle\mathbf{a}\rangle \text {, cleavage }\end{array}$ \\
\hline $\begin{array}{l}\text { Pyramidal I } \\
\text { Pyramidal II }\end{array}$ & $\begin{array}{l}(10 \overline{1} 0)[1 \overline{2} 10] \\
(11 \overline{2} 2)[\overline{1} 010]\end{array}$ & $\begin{array}{l}\text { basal } \\
\text { basal }\end{array}$ & $\begin{array}{l}767 \\
647\end{array}$ & $\begin{array}{l}125 \\
125\end{array}$ & $\begin{array}{l}0.292 \\
0.269\end{array}$ & $\begin{array}{l}0.222 \\
0.250\end{array}$ & $\begin{array}{l}\text { basal }\langle\mathbf{a}\rangle \\
\text { basal }\langle\mathbf{a}\rangle\end{array}$ & $\begin{array}{l}\text { basal }\langle\mathbf{a}\rangle \\
\text { cleavage }\end{array}$ & $\begin{array}{l}\text { basal }\langle\mathbf{a}\rangle \\
\text { basal }\langle\mathbf{a}\rangle\end{array}$ & $\begin{array}{l}\text { basal }\langle\mathbf{a}\rangle \\
\text { basal }\langle\mathbf{a}\rangle\end{array}$ \\
\hline Twin & $\begin{array}{l}(10 \overline{1} 2)[\overline{1} 2 \overline{1} 0] \\
(10 \overline{1} 2)[1 \overline{2} 10]\end{array}$ & $\begin{array}{l}\text { basal } \\
\text { basal }\end{array}$ & 661 & 125 & 0.257 & $\begin{array}{l}0.279 \\
0.743\end{array}$ & $\begin{array}{l}\text { cleavage } \\
\text { cleavage }\end{array}$ & $\begin{array}{l}\text { cleavage } \\
\text { cleavage }\end{array}$ & $\begin{array}{l}\text { cleavage } \\
\text { cleavage }\end{array}$ & $\begin{array}{l}\text { cleavage } \\
\text { cleavage }\end{array}$ \\
\hline basal-prismatic & {$[1 \overline{2} 10]$} & basal & 575 & 125 & 0.229 & 0.236 & cleavage & cleavage & cleavage & cleavage \\
\hline
\end{tabular}

Table 1: Crack tip cleavage/emission competition in magnesium as predicted from linear elastic fracture mechanics and as observed in atomistic simulations using a modified embedded-atom method interatomic potential. (n) is the crack plane and $[\mathbf{l}]$ is the crack front direction. For cases with multiple dislocation slip systems, the slip system with the lowest $K_{I e}$ is shown. Interface energy $\gamma_{i}$ (relaxed) for twin and basal-prismatic interfaces are 148 and $211 \mathrm{~mJ} \mathrm{~m}^{-2}$, respectively.

Examining Table 1, we see that for the basal plane cracks, oriented for $\langle\mathbf{c}+\mathbf{a}\rangle$ dislocation emission on pyramidal I and II planes, respectively, the $K_{I e}$ values are much higher $\left(38 \%\right.$ and $31 \%$ ) than $K_{I c}$, suggesting cleavage will occur without any dislocation emission. For the crack on the pyramidal I plane, $K_{I e}$ for dislocation emission on the basal planes is substantially lower (24\%) than $K_{I c}$, and so dislocation emission is predicted in this case. For the cracks on prismatic planes and the pyramidal II planes, $K_{I e}$ is marginally lower than $K_{I c}$. Finally, $K_{I c}$ is lower than $\mathrm{K}_{I e}$ in all three interface cracks. The crack on the twin interface in the brittle orientation has $K_{I e}$ nearly 3 times of its $K_{I c}$. The above predictions of $K_{I c}$ and $K_{I e}$ suggest that $\mathrm{Mg}$ is rather brittle, being prone to Griffith cleavage in many orientations: out of the 9 cracks considered, 3 orientations clearly favor Griffith cleavage, 2 orientations marginally favor Griffith cleavage, 3 orientations marginally favor dislocation emission, and only 1 orientation clearly favors dislocation emission. The crack on the BP interface has the lowest $K_{I c}$ and cracks 
on basal plane, one of the prismatic plane and twin interface have nearly identical $K_{I c}$. Overall, $K_{I c}$ for cleavage on different crystallographic planes are not widely different among all the cases.

The theory shows a number of cases where the competition is very close, i.e. $K_{I c} \approx K_{I e}$. Definitive predictions are thus not possible because of the implicit approximations in the Rice theory, the discreteness of the atomic system (especially for interface cracks where some lattice trapping may exist due to the microscope variations of surface energy), and the assumption of linear elasticity. While the theory has previously been shown to agree fairly well with simulations on a range of crack-tip phenomena $[4,5,6,31]$, simulations remain essential for validating the theory, and we now report the simulation results.

Fig. 3 shows the cross-section of the atomistic crack tip regions in a plane perpendicular to the crack line at different applied $K_{I}$ values for initial sharp cracks. In each case, the top picture shows the initial crack tip structure determined from the anisotropic elastic solution at a $K_{I}$ slightly below $K_{I c}$, the middle picture shows the relaxed structure at the initial $K_{I}$, and the bottom picture shows the crack tip structure at a higher applied $K_{I}$ load after cleavage or emission has started. During each relaxation step, the boundary conditions are applied based on the initial crack tip position and the $K_{I c}$ values are determined to be within a small range $(<5 \%$ of the mean value between crack advance and closure) in the simulations.

Fig. 3 (a) and (b) show the basal plane cracks where both cracks exhibit Griffith cleavage behavior under loads near their $K_{I c}$ value; the crack closes at loads slightly below its $K_{I c}$ and advances at loads slightly higher than its $K_{I c}$. This is also consistent with the LEFM prediction where $K_{I e}$ for nucleating the first $\langle\mathbf{c}+\mathbf{a}\rangle$ partial dislocation is substantially higher than $K_{I c}$. Fig. 3 (c), (d) and (f) show the two cracks on prismatic planes and a crack on pyramidal II plane. In these three cases, cleavage is observed despite their $K_{I c}$ values being 7\%, 2\%, and $8 \%$ higher than their respective $K_{I e}$ for dislocation emission. In all these 5 cases, no appreciable lattice trapping is observed.

The crack on the pyramidal I plane (Fig. 3 (e)) shows dislocation emission immediately following relaxation. In this case, $K_{I c}$ is $32 \%$ higher than the $K_{I e}$, well above the loading for nucleating a leading partial dislocation on the basal plane. After nucleation, the partial dislocation glides away from the crack tip, leaving behind a stacking fault. This process also creates a step, which blunts and stabilizes the crack tip against closure at this load below $K_{I c}$. Upon increasing the applied load, a second partial dislocation with the same Burgers vector as the first one is nucleated, further blunting the crack tip. The process of dislocation 
This is a pre-print of the following article: Wu, Z.; Curtin, W. A. Acta Mater. 2015, 88, 1-12.. The formal publication is available at http://dx.doi.org/10.1016/j.actamat.2015.01.023

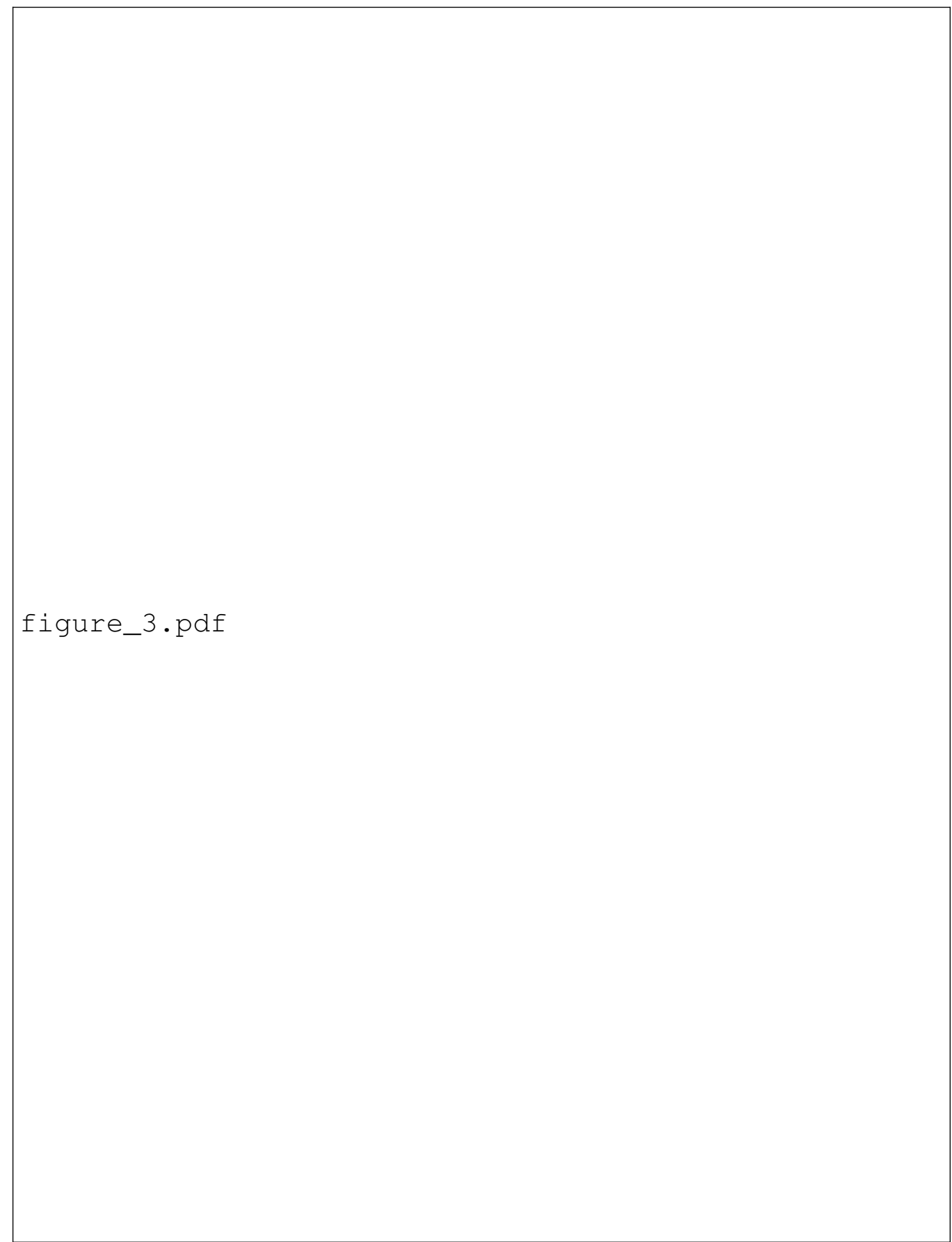

Figure 3: Cross-sectional view of sharp cracks corresponding to cases in Fig. 2. Atoms in this figure (and Fig. 4 and 5) are colored based on Common Neighbor Analysis [32]; blue for hcp, green for fcc, orange for bcc and red for others. 
nucleation continues with higher applied loads; this crack tip thus exhibits ductile behavior.

Fig. 3 (g) and (h) show the two tensile twin interface cracks. In these two cases, the crack surface is placed slightly above the twin interface. Upon relaxation, the lower crack surface undergoes substantial reconstruction from its original flat, twin interface structure. This relaxation process also modifies the crack tip structure slightly and prevents the crack from closing, even though it is loaded below $K_{I c}$. For the first twin interface crack in the presumed "ductile" orientation, cleavage occurs with crack propagating along the twin interface at an applied load of $1.21 K_{I c}$. The resulting crack surfaces adopt a zig-zag form alternating between basal and pyramidal I planes. Comparing to the previous cracks in single crystals where cleavages occur nearly at the predicted $K_{I c}$ values (within 3-6\%), the crack at the twin interface cleaves at a load $21 \%$ higher than the $K_{I c}$ predicted based on $\gamma_{s}$ of the $(10 \overline{1} 2)$ plane and $\gamma_{i}$ of the twin boundary. This cleavage load is also $11 \%$ higher than the predicted $K_{I e}$ and yet no dislocation is emitted. The interface crack in the presumed "brittle" orientation undergoes some atomic reconstruction and blunting process at the crack tip with increasing load, which makes it resist cleavage well above the predicted $K_{I c}$. At some higher load level, a sharp crack is nucleated and propagates along a prismatic plane. In both cases, cleavage is the dominant failure mechanism. Finally, Fig. 3 (i) shows the crack at the BP interface which adopts a semi-coherent structure varying periodically along the boundary. The crack surface also undergoes surface reconstruction upon relaxation, blunting the crack tip slightly and preventing crack closure, but cleavage fracture eventually occurs at a load $20 \%$ higher than the $K_{I c}$ predicted based on the average BP interface energy.

Both the LEFM analysis and the atomistic simulations show that atomically sharp cracks undergo cleavage in many of the cases considered. Furthermore, the three cases predicted to slightly favor dislocation emission show cleavage behavior in atomistic simulations. These results suggest that $\mathrm{Mg}$ has very low intrinsic fracture toughness and is prone to brittle cleavage failure since initially sharp cracks stay sharp during cleavage. The atomically-sharp crack geometry can seem idealized. However, even if initial defects are blunted, if crack growth can be initiated then the sharp crack result is relevant in determining whether crack growth via cleavage will be sustained or whether the sharp crack will be blunted. Thus, the sharp-crack result provides a fundamental baseline assessment of brittle vs ductile behavior in a material.

Sharp propagation cracks could be blunted by other mechanisms, not just dislocation emission from crack tips. For example, cracks may cut through pre- 
existing defects or dislocations during their propagation. Slightly blunted crack tips can change the elastic field around the crack tips, and thus may shift the competition between Griffith cleavage and dislocation emission, as is well recognized and studies [33, 34]. For completeness, we have thus examined slightly blunted cracks to determine the robustness of the conclusions obtained above for sharp cracks.

Fig. 4 and 5 show the results of blunted cracks with tip radius of $\sim 0.5 \mathrm{~nm}$ and $1 \mathrm{~nm}$, respectively. The crack tips shown in these figures are all relaxed structures. In the first basal plane crack, as shown in Fig. 4 (a), a defect resembling a leading $\langle\mathbf{c}+\mathbf{a}\rangle$ partial dislocation on pyramidal I plane is nucleated at a load of $1.45 \mathrm{~K}_{\text {Ic }}$. This load is also very close to the predicted $K_{I e}$ for such dislocation nucleation. However, this initial defect does not evolve into a proper dislocation able to glide away from the crack tip. With increasing applied load, an atomically sharp basal plane crack is nucleated near this defect and cleavage becomes dominant along this newly nucleated crack. This crack behaves similarly for the larger crack tip radius (Fig. 5 (a)) where the crack tip undergoes substantial reconstruction and local defect nucleation, but cleaves finally. For this crack orientation, initial crack tip blunting does not change its cleavage behavior. Fig. 4 (b) shows the other basal plane crack where a full $\langle\mathbf{c}+\mathbf{a}\rangle$ dislocation (leading partial followed by its trailing partial) is emitted on the pyramidal II plane at a load of $1.41 K_{I c}$, close to its predicted $K_{I e}$. The $\langle\mathbf{c}+\mathbf{a}\rangle$ dislocation glides away, changing the crack tip geometry, which in turn resharpens the crack tip. As a result, a new atomically sharp crack is nucleated and cleaves as in the previous atomically sharp crack. Fig. 5 (b) shows the crack with the crack tip radius increased to $\sim 1 \mathrm{~nm}$. In this crack, more $\langle\mathbf{c}+\mathbf{a}\rangle$ dislocations are emitted with increasing the applied loads. However, dislocation plasticity in the early stage resharpens the blunted crack tip, resulting in new sharp crack being nucleated and its final cleavage. In this crack orientation, the blunted crack tip causes very limited dislocation emissions and does not change the dominant cleavage behavior.

Fig. 4 (c) shows the crack on the first prismatic plane with crack tip radius of $\sim 0.5 \mathrm{~nm}$. At a load of $1.07 K_{I c}$, an atomically sharp crack is nucleated and propagates, as in the sharp crack case and with only a minor increase in load. For the crack with tip radius of $\sim 1 \mathrm{~nm}$ (see Fig. 5 (c)), a pair of partial dislocations on basal planes are nucleated at different locations near, but not exactly at, the crack tip. This occurs at an applied load of $1.33 K_{I c}$, which is much higher than those in the previous sharp and slightly blunted cases. The crack tip is also resharpened at one of the dislocation nucleation site, which leads to an atomically sharp crack being nucleated immediately after the dislocation glides away (Fig. 5 (c2)) 
This is a pre-print of the following article: Wu, Z.; Curtin, W. A. Acta Mater. 2015, 88, 1-12.. The formal publication is available at http://dx.doi.org/10.1016/j.actamat.2015.01.023

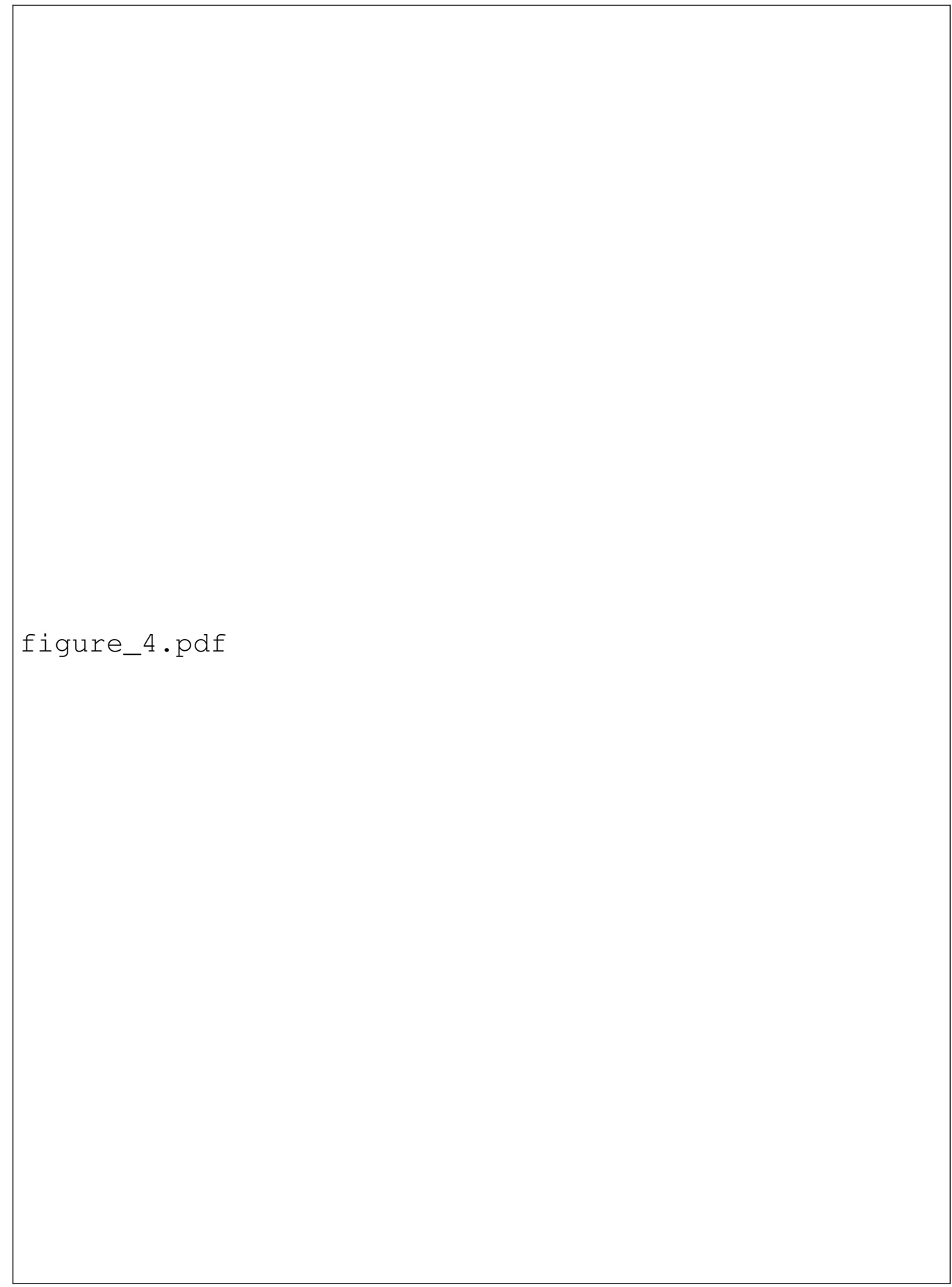

Figure 4: Cross-sectional view of slightly blunted cracks with tip radius $\sim 0.5 \mathrm{~nm}$. 
This is a pre-print of the following article: Wu, Z.; Curtin, W. A. Acta Mater. 2015, 88, 1-12.. The formal publication is available at http://dx.doi.org/10.1016/j.actamat.2015.01.023

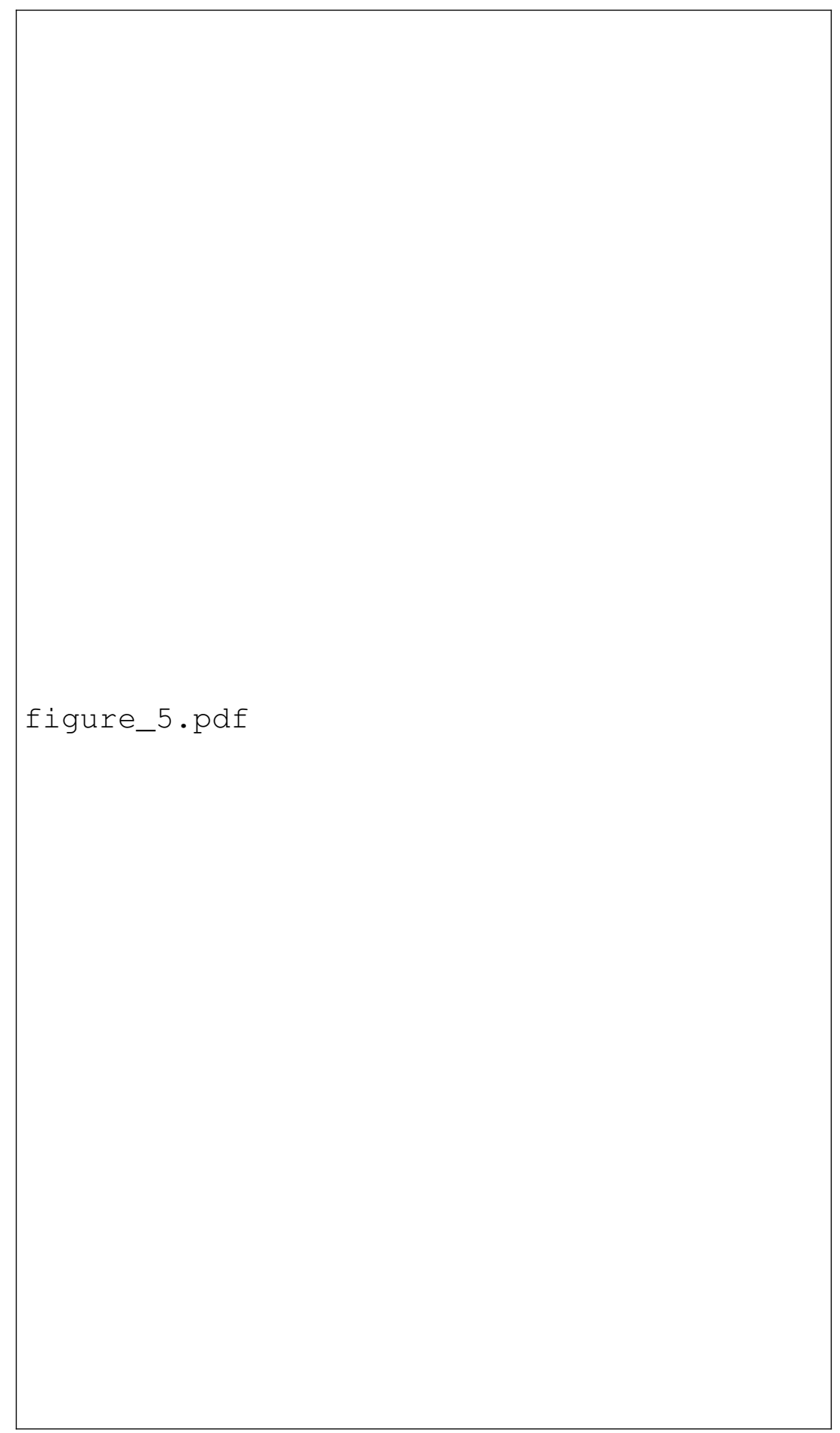

Figure 5: Cross-sectional view of slightly blunted cracks with tip radius $\sim 1 \mathrm{~nm}$. 17 
followed by cleavage. In both cases of blunted crack tip, the response is brittle even though a couple of dislocations are emitted. Fig. 4 (d) shows the second crack on the prismatic plane. Initially, a leading partial dislocation on the basal plane is nucleated at the crack tip at a load of $1.12 K_{I c}$, marginally higher than the predicted $K_{I e}$ for sharp cracks. The newly nucleated dislocation glides upward, leaving behind a stacking fault and a sharp tip of one atomic plane thickness at the dislocation nucleation site. With the applied load increased to $1.27 K_{I c}$, a second partial dislocation on basal plane is nucleated on the adjacent basal plane, which further sharpens the crack tip and leads to a new sharp crack being nucleated and propagating in a cleavage mode. The crack with increased tip radius of $\sim 1 \mathrm{~nm}$ behaves similarly (Fig. $5(\mathrm{~d})$ ): at a load of $1.24 K_{I c}$, a pair of partial dislocations on basal planes are nucleated simultaneously and glide in opposite directions. With increasing the applied load to $1.42 K_{I c}$, a sharp crack is nucleated and propagates brittlely. We note that during further tests (not shown here) on cracks with slightly varied tip geometry and/or radius, the crack can exhibit a ductile behavior when the crack tip is constructed with a type of special configuration allowing for continuous simultaneous dislocation nucleation in opposite directions. The symmetrical event does not change the crack tip geometry and so with increasing applied loads, the dislocation nucleation and glide process continues, and the response is ductile. Nevertheless, the ductile behavior is only seen in a case of special tip configuration and at finite temperatures, simultaneous nucleation is not likely. We therefore expect brittle cleavage to be dominant for this crack orientation.

Fig. 4 (e) and 5 (e) show the cracks on the pyramidal I plane with tip radius of $\sim 0.5$ and $1 \mathrm{~nm}$. In these two cases, dislocations are first nucleated at loads of 0.99 and $1.06 K_{I c}$, similar to that in the atomically sharp crack. The blunted crack tip has little effect on the crack behavior; the crack behaves in a ductile manner with continuous dislocation nucleation in all three crack tip configurations. The crack on pyramidal II plane with crack tip radius of $\sim 0.5$ and $1 \mathrm{~nm}$ are shown in Fig. 4 (f) and 5 (f). In this crack orientation, dislocations are first nucleated at 1.00 and $1.08 K_{I c}, 2 \%$ and $10 \%$ higher than the predicted $K_{I e}$ for sharp cracks. Both cracks show continuous dislocation emission with increasing loads, in contrast to the brittle cleavage behavior exhibited in the sharp crack simulation. In this case, the slightly blunted crack tip shifts the competition from cleavage to dislocation emission for this crack orientation.

Fig. $4(\mathrm{~g})$ and $5(\mathrm{~g})$ show the blunted twin interface cracks in the "ductile" orientation. In both cases, substantial atomic reconstructions are seen at the crack tips upon relaxation. Such reconstructions not only change the crack tip geometry and lattice, but also shift the ideal twin interface structure. With increasing 
loads, sharp cracks are nucleated at the crack tips, which eventually leads to brittle cleavages along the twin interfaces. This cleavage process is similar to that in the sharp crack case, but occurs at loads of 1.60 and $1.63 K_{I c}$, substantially higher than $1.21 K_{I c}$ seen in the sharp crack case. In this crack orientation, the tip bluntness increases the load for cleavage, but does not change the crack tip competition. Fig. 4 (h) and 5 (h) show the blunted twin interface cracks in the "brittle" orientation. Similar to the previous cracks in the ductile orientation, substantial atomic reconstructions occur at the crack tips. For the crack with tip radius of $\sim 0.5 \mathrm{~nm}$ (Fig. 4 (h1)), a partial dislocation on a basal plane is nucleated at the crack tip and glides upward towards the twin interface. While the dislocation nucleation process creates an atom size sharp crack, the dislocation reaction at the twin interface shifts the twin boundary and creates a short segment of BP interface boundary. At a higher applied load of $1.18 K_{I c}$ (see Fig. 4 (h2)), the crack cleaves along the newly formed BP interface segment and is arrested at the end of the BP segment. At the new crack tip, a partial dislocation on basal plane is nucleated and glides downward towards the twin interface, creating a segment of $\mathrm{BP}$ interface and shifting the twin interface, as for the first partial dislocation. The crack tip structure is restored to the initial structure and at a higher load, cleavage occurs along the short BP boundary segment and a partial dislocation is again nucleated. This process is repeated as the crack grows in a zig-zag form, alternating between basal and prismatic surfaces but with an average growth orientation still along the twin interface. Interestingly, the applied load for cleavage is $1.32 K_{I c}$, much lower than the value of $1.63 K_{I c}$ in the sharp crack. In the case of the $\sim 1 \mathrm{~nm}$ crack tip radius, the crack behaves similarly to the sharp crack case. The crack tip undergoes substantial reconstruction with increasing loads until a new sharp crack is nucleated along the prismatic plane in the upper crystal, followed by cleavage. In this crack orientation, the cleavage plane and the cleavage load are again very sensitive to the crack tip geometry and its subsequent reconstruction but cleavage remains the dominant mechanism in all three crack tip configurations. Finally, Fig. 4 (i) and 5 (i) show the BP interface cracks with blunted tips. In both cases, the cracks behave similarly to the sharp crack, cleaving along the BP interface at similar stress intensity factors. The crack tip bluntness has little effect on the crack tip response.

Comparisons between all the blunted cracks with their respective sharp crack show that when the predicted $K_{I e}$ is close to $K_{I c}$, slight crack tip bluntness may help initiate early dislocation emission, but does not change the dominant crack tip behavior in general. The only exception is the crack on pyramidal II plane which changes its dominant crack tip behavior from Griffith cleavage to disloca- 
tion emission. In case (c), (e) and (i), crack tip bluntness has little effects on the crack behavior. In other cases, slightly blunting the crack tip increases its cleavage load so that sharp cracks can be nucleated through limited dislocation emission (case (b) and (d)) or crack tip reconstructions (case (a), (g) and (h)). Overall, the intrinsic fracture property of $\mathrm{Mg}$ revealed in the sharp crack study remains; it has low intrinsic fracture toughness in most orientations and is susceptible to Griffith cleavage.

\section{Discussion and conclusion}

We have presented a systematic investigation on the crack tip competition between dislocation emission and Griffith cleavage in Mg using both LEFM analysis and atomistic simulations. For sharp cracks, the LEFM predictions are consistent with atomistic simulations in cases where the $K_{I c}$ and $K_{I e}$ values are well separated. In three cases where $K_{I e}$ values are marginally lower $(<10 \%)$ than $K_{I e}$ values, atomistic simulations show brittle cleavage, defying the dislocation emission prediction. This is not particularly surprising given the assumptions and approximations employed in the LEFM analysis and the discreteness of the atomic system. For all the cracks in single crystal, the predicted $K_{I c}$ value for Griffith cleavage agrees remarkably well with that in atomistic simulations. For interface cracks, the predicted $K_{I c}$ show some deviations from that in simulations due to crack tip reconstructions and local plasticity, and microscopic structure variations along interface boundaries. Nevertheless, the dominant plastic mechanisms in simulations still agree with the predictions. Overall, both the LEFM analysis and atomistic simulations show the dominant brittle cleavage behavior of $\mathrm{Mg}$ and its low intrinsic fracture toughness at most of the sharp cracks.

Initial slight crack tip bluntness has limited effects on changing the crack tip behavior. For basal cracks, $\langle\mathbf{c}+\mathbf{a}\rangle$ dislocations are initially emitted from the blunted crack tip in one of the case. However, the initial dislocation plasticity is very limited and more importantly, it resharpens the crack tip, leading to cleavage fracture at higher loads. Similar behavior is observed for prismatic plane cracks. We can thus loosely categorize these basal and prismatic plane cracks as intrinsically brittle, despite some limited dislocations initially emitted in slightly blunted cracks. Cracks on pyramidal I planes are intrinsically ductile as they continuously emit dislocations regardless of crack tip geometry. Cracks on pyramidal II planes cleave in the sharp crack tip case and emit dislocations with slightly blunted crack tips, suggesting their quasi-brittle nature. In the twin interface cracks, the crack tips undergo substantial reconstructions. The atomic crack tip geometry also has 
some effects in changing their cleavage loads and cleavage planes. Nevertheless, cleavage is the dominant mechanism for both crack orientations with sharp and blunted crack tips. This also suggests twin boundaries are intrinsically brittle and can be damage initiation sites. The BP interface cracks are intrinsically brittle as well, as they cleave regardless of the initial crack tip geometry. All three interface cracks show brittle cleavage behavior.

Examining all the 9 crack orientations and their respective slip systems in the current study shows that the basal plane cracks have the $\langle\mathbf{c}+\mathbf{a}\rangle$ dislocation slip system competing against cleavage while for the rest, the $\langle\mathbf{a}\rangle$ dislocation slip system on the basal plane is competing against cleavage since it has the lowest $K_{I e}$. Therefore, $\langle\mathbf{c}+\mathbf{a}\rangle$ dislocation emission at basal crack tips is crucial to prevent basal cleavage. The LEFM analysis suggests that, to activate the $\langle\mathbf{c}+\mathbf{a}\rangle$ dislocation slip system, a significant reduction of its $\gamma_{u s}$, on the order of $50 \%$, is required if other properties are kept unchanged. In other crack orientations, except the twin interface crack in the "brittle" orientation, a moderate reduction $(\sim 20 \%)$ of $\gamma_{u s}$ on the basal plane is sufficient to shift the competition to favor dislocation emission. The reductions of $\gamma_{u s}$ can probably be approached through alloying but their relatively large magnitudes may pose significant challenges.

In summary, we have studies the crack tip competitions in $\mathrm{Mg}$ for a broad range of crack orientations. Both the LEFM prediction and atomistic simulations show that $\mathrm{Mg}$ is essentially intrinsically brittle because crack tip dislocation nucleation is not observed in almost all geometries where it would be most favorable. This demonstrates that toughening in $\mathrm{Mg}$ might emerge only from two aspects: (i) possible dislocation nucleation at finite temperatures due to thermal activation, which is, however, not assured given the substantial reductions of $\gamma_{u s}$ required, and (ii) extrinsic plasticity in the surrounding material, which provides some toughening but not the levels associated with ductile rupture that can be achieved via crack tip blunting by dislocation nucleation. Our results are also consistent with low temperature experimental observations, and suggest that $\mathrm{Mg}$ fracture is more likely at higher loading rates, such as occurs in crash loading. Nevertheless, we emphasize that the analysis and simulations here are based on properties at $0 \mathrm{~K}$ temperature. Material properties, such as the elastic constants, surface free energies and the unstable stacking fault free energies, change with temperatures. The thermal effects on the crack tip competition, crack nucleation, as well as extrinsic effects such as preexisting dislocations and grain boundaries, require further investigations and will be the subject of our future work. 


\section{Acknowledgements}

ZW gratefully acknowledges the financial support from the Agency for Science, Technology and Research (A*STAR), Singapore and the use of computing resources at the A*STAR Computational Resource Centre, Singapore. WAC acknowledges support of this work through a European Research Council Advanced Grant, "Predictive Computational Metallurgy", ERC Grant agreement No. 339081 - PreCoMet.

\section{Appendix}

For 2D plane elasticity problems with $u_{k, 3}=0$, static equilibrium condition gives

$$
\sigma_{i j, j}=C_{i j k l} u_{k, l j}=0
$$

The above equation is a second order differential equation in $x_{1}$ and $x_{2}$. The general solution is

$$
u_{k}=a_{k} f(z), \text { i.e., } \mathbf{u}=\mathbf{a} f(z)
$$

where

$$
z=x_{1}+p x_{2}
$$

and $f$ is an arbitrary function of $z . p$ and a are constants satisfying

$$
\left[\mathbf{Q}+p\left(\mathbf{R}+\mathbf{R}^{T}\right)+p^{2} \mathbf{T}\right] \mathbf{a}=\mathbf{0}
$$

where

$$
Q_{i k}=C_{i 1 k 1}, R_{i k}=C_{i 1 k 2}, T_{i k}=C_{i 2 k 2}
$$

For nontrivial solutions of the eigenvalue $p$ and eigenvector a,

$$
\left|\mathbf{Q}+p\left(\mathbf{R}+\mathbf{R}^{T}\right)+p^{2} \mathbf{T}\right|=0
$$

Eqn. A.6 is solved to obtain six roots for the eigenvalue $p_{\alpha}$ and corresponding eigenvector $\mathbf{a}_{\alpha}$,

$$
\operatorname{Im}\left(p_{\alpha}\right)>0, p_{\alpha+3}=\bar{p}_{\alpha}, \mathbf{a}_{\alpha+3}=\overline{\mathbf{a}}_{\alpha}, \alpha=1,2,3
$$

In cases where all the eigenvalues are distinct, the general solution is obtained by superposition of the six solutions, i.e.,

$$
\mathbf{u}=\sum_{\alpha=1}^{3}\left\{\mathbf{a}_{\alpha} f_{\alpha}\left(z_{\alpha}\right)+\overline{\mathbf{a}}_{\alpha+3} f_{\alpha}\left(\bar{z}_{\alpha}\right)\right\}
$$


and the general solution for stresses can be expressed by a stress function

$$
\boldsymbol{\psi}=\sum_{\alpha=1}^{3}\left\{\mathbf{b}_{\alpha} f_{\alpha}\left(z_{\alpha}\right)+\overline{\mathbf{b}}_{\alpha} f_{\alpha+3}\left(\bar{z}_{\alpha}\right)\right\}
$$

where

$$
\mathbf{b}_{\alpha}=\left(\mathbf{R}^{T}+p_{\alpha} \mathbf{T}\right) \mathbf{a}_{\alpha}=-\frac{1}{p_{\alpha}}\left(\mathbf{Q}+p_{\alpha} \mathbf{R}\right) \mathbf{a}_{\alpha}
$$

and

$$
\mathbf{b}_{\alpha+3}=\overline{\mathbf{b}}_{\alpha}, \alpha=1,2,3
$$

The arbitrary function $f_{\alpha}(z)$ often takes the same function form as

$$
f_{\alpha}\left(z_{\alpha}\right)=f\left(z_{\alpha}\right) q_{\alpha} \text { and } f_{\alpha+3}\left(\bar{z}_{\alpha}\right)=\bar{f}\left(\bar{z}_{\alpha}\right) \bar{q}_{\alpha}
$$

therefore the elastic field solution can be written as

$$
\mathbf{u}=2 \operatorname{Re}\left\{\mathbf{A}\left\langle f\left(z_{\alpha}\right)\right\rangle \mathbf{q}\right\}, \text { and } \boldsymbol{\psi}=2 \operatorname{Re}\left\{\mathbf{B}\left\langle f\left(z_{\alpha}\right)\right\rangle \mathbf{q}\right\}
$$

where

$$
\mathbf{A}=\left[\mathbf{a}_{1}, \mathbf{a}_{2}, \mathbf{a}_{3}\right], \text { and } \mathbf{B}=\left[\mathbf{b}_{1}, \mathbf{b}_{2}, \mathbf{b}_{3}\right],
$$

with the eigenvectors $\mathbf{a}$ and $\mathbf{b}$ normalized such that

$$
\mathbf{b}_{\alpha}^{T} \mathbf{a}_{\beta}+\mathbf{a}_{\alpha}^{T} \mathbf{b}_{\beta}=\delta_{\alpha \beta}, \delta_{\alpha \beta} \text { is the Kronecker delta }
$$

and

$$
\left\langle f\left(z_{\alpha}\right)\right\rangle=\operatorname{diag}\left[f\left(z_{1}\right), f\left(z_{2}\right), f\left(z_{3}\right)\right]
$$

the unknown function $f\left(z_{\alpha}\right)$ and complex vector $\mathbf{q}$ are determined from the boundary conditions. For a crack of length $2 a$ in an infinite homogeneous medium subject to uniform traction $\mathbf{t}_{\Gamma}$ and $-\mathbf{t}_{\Gamma}$ applied on the $\pm x_{2}$ surfaces at infinity, the elastic field solution is given as Eqn. 1 in Section 2.

\section{References}

[1] A. A. Griffith, The phenomena of rupture and flow in solids, Philosophical Transactions of the Royal Society of London. Series A, Containing Papers of a Mathematical or Physical Character 221 (1921) 163-198.

[2] J. R. Rice, Dislocation nucleation from a crack tip: An analysis based on the peierls concept, Journal of the Mechanics and Physics of Solids 40 (1992) 239-271. 
[3] E. B. Tadmor, S. Hai, A peierls criterion for the onset of deformation twinning at a crack tip, Journal of the Mechanics and Physics of Solids 51 (2003) $765-793.1$.

[4] S. Hai, E. B. Tadmor, Deformation twinning at aluminum crack tips, Acta Materialia 51 (2003) 117-131.

[5] D. H. Warner, W. A. Curtin, S. Qu, Rate dependence of crack-tip processes predicts twinning trends in f.c.c. metals, Nature Materials 6 (2007) 876-881. 10.1038/nmat2030.

[6] D. H. Warner, W. A. Curtin, Origins and implications of temperaturedependent activation energy barriers for dislocation nucleation in facecentered cubic metals, Acta Materialia 57 (2009) 4267-4277.

[7] B. Bhattacharya, M. Niewczas, Work-hardening behaviour of mg single crystals oriented for basal slip, Philosophical Magazine 91 (2011) 22272247.

[8] J. A. Yasi, T. Nogaret, D. R. Trinkle, Y. Qi, L. G. H. Jr, W. A. Curtin, Basal and prism dislocation cores in magnesium: comparison of first-principles and embedded-atom-potential methods predictions, Modelling and Simulation in Materials Science and Engineering 17 (2009) 055012.

[9] J.-J. Tang, X.-B. Yang, L. OuYang, M. Zhu, Y.-J. Zhao, A systematic firstprinciples study of surface energies, surface relaxation and friedel oscillation of magnesium surfaces, Journal of Physics D: Applied Physics 47 (2014) 115305.

[10] M. Ghazisaeidi, L. G. Hector Jr, W. A. Curtin, First-principles core structures of edge and screw dislocations in mg, Scripta Materialia 75 (2014) $42-45$.

[11] Z. Wu, M. F. Francis, W. A. Curtin, Magnesium interatomic potential for simulating plasticity and fracture phenomena, Modelling and Simulation in Materials Science and Engineering accepted (2014).

[12] T. C. Ting, Anisotropic Elasticity: Theory and Applications, Oxford University Press, 1996. 
[13] J. Qu, J. L. Bassani, Cracks on bimaterial and bicrystal interfaces, Journal of the Mechanics and Physics of Solids 37 (1989) 417-433.

[14] J. L. Bassani, J. Qu, Finite crack on bimaterial and bicrystal interfaces, Journal of the Mechanics and Physics of Solids 37 (1989) 435-453.

[15] Y. Sun, G. E. Beltz, Dislocation nucleation from a crack tip: A formulation based on anisotropic elasticity, Journal of the Mechanics and Physics of Solids 42 (1994) 1905-1932.

[16] B. Kondori, A. A. Benzerga, Effect of stress triaxiality on the flow and fracture of mg alloy az31, Metallurgical and Materials Transactions A Physical Metallurgy and Materials Science 45A (2014) 3292-3307.

[17] V. Kaushik, R. Narasimhan, R. K. Mishra, Experimental study of fracture behavior of magnesium single crystals, Materials Science and Engineering: A 590 (2014) 174-185.

[18] Y. Cheng, Z.-H. Jin, Y. Zhang, H. Gao, On intrinsic brittleness and ductility of intergranular fracture along symmetrical tilt grain boundaries in copper, Acta Materialia 58 (2010) 2293-2299.

[19] J. Wang, R. G. Hoagland, J. P. Hirth, L. Capolungo, I. J. Beyerlein, C. N. Tomé, Nucleation of a (1012) twin in hexagonal close-packed crystals, Scripta Materialia 61 (2009) 903-906.

[20] A. Luque, M. Ghazisaeidi, W. A. Curtin, Deformation modes in magnesium (0001) and (0111) single crystals: simulations versus experiments, Modelling and Simulation in Materials Science and Engineering 21 (2013) 045010 .

[21] B. Xu, L. Capolungo, D. Rodney, On the importance of prismatic/basal interfaces in the growth of twins in hexagonal close packed crystals, Scripta Materialia 68 (2013) 901-904.

[22] A. Ostapovets, R. Gröger, Twinning disconnections and basal-prismatic twin boundary in magnesium, Modelling and Simulation in Materials Science and Engineering 22 (2014) 025015.

[23] C. D. Barrett, H. El Kadiri, Impact of deformation faceting on and embryonic twin nucleation in hexagonal close-packed metals, Acta Materialia 70 (2014) 137-161. 
[24] J. Wang, L. Liu, C. N. Tomé, S. X. Mao, S. K. Gong, Twinning and detwinning via glide and climb of twinning dislocations along serrated coherent twin boundaries in hexagonal-close-packed metals, Materials Research Letters 1 (2013) 81-88.

[25] B.-Y. Liu, J. Wang, B. Li, L. Lu, X.-Y. Zhang, Z.-W. Shan, J. Li, C.-L. Jia, J. Sun, E. Ma, Twinning-like lattice reorientation without a crystallographic twinning plane, Nat Commun 5 (2014) 3297.

[26] S. Plimpton, Fast parallel algorithms for short-range molecular dynamics, Journal of Computational Physics 117 (1995) 1-19.

[27] A. Stukowski, Visualization and analysis of atomistic simulation data with ovito-the open visualization tool, Modelling and Simulation in Materials Science and Engineering 18 (2010) 015012.

[28] M. I. Baskes, Modified embedded-atom potentials for cubic materials and impurities, Physical Review B 46 (1992) 2727-2742.

[29] B.-J. Lee, M. I. Baskes, Second nearest-neighbor modified embedded-atommethod potential, Physical Review B 62 (2000) 8564-8567.

[30] Y.-M. Kim, N. J. Kim, B.-J. Lee, Atomistic modeling of pure mg and mg-al systems, Calphad 33 (2009) 650-657.

[31] J. Song, W. A. Curtin, A nanoscale mechanism of hydrogen embrittlement in metals, Acta Materialia 59 (2011) 1557-1569.

[32] D. Faken, H. Jónsson, Systematic analysis of local atomic structure combined with 3d computer graphics, Computational Materials Science 2 (1994) 279-286.

[33] L. L. Fischer, G. E. Beltz, The effect of crack blunting on the competition between dislocation nucleation and cleavage, Journal of the Mechanics and Physics of Solids 49 (2001) 635-654.

[34] M. Huang, Z. Li, Dislocation emission criterion from a blunt crack tip, Journal of the Mechanics and Physics of Solids 52 (2004) 1991-2003. 